\title{
First Law of Holographic Complexity
}

\author{
Alice Bernamonti, ${ }^{1,2,{ }^{*}}$ Federico Galli, ${ }^{1, \dagger}$ Juan Hernandez, ${ }^{1,3, \$}$ Robert C. Myers, ${ }^{1, \S}$ Shan-Ming Ruan, ${ }^{1,3, \|}$ and Joan Simón ${ }^{4, \uparrow}$ \\ ${ }^{1}$ Perimeter Institute for Theoretical Physics, Waterloo, Ontario N2L 2Y5, Canada \\ ${ }^{2}$ Dipartimento di Fisica, Università di Firenze and INFN Sezione di Firenze, Via G. Sansone 1, 50019 Sesto Fiorentino, Italy \\ ${ }^{3}$ Department of Physics and Astronomy, University of Waterloo, Waterloo, Ontario N2L 3G1, Canada \\ ${ }^{4}$ School of Mathematics and Maxwell Institute for Mathematical Sciences, University of Edinburgh, \\ Edinburgh EH9 3FD, United Kingdom
}

(Received 27 March 2019; published 22 August 2019)

\begin{abstract}
We investigate the variation of holographic complexity for two nearby target states. Based on Nielsen's geometric approach, we find the variation only depends on the end point of the optimal trajectory, a result which we designate the first law of complexity. As an example, we examine the complexity = action conjecture when the anti-de Sitter vacuum is perturbed by a scalar field excitation, which corresponds to a coherent state. Remarkably, the gravitational contributions completely cancel and the final variation reduces to a boundary term coming entirely from the scalar field action. Hence, the null boundary of Wheeler-DeWitt patch appears to act like the "end of the quantum circuit".
\end{abstract}

DOI: 10.1103/PhysRevLett.123.081601

Introduction.-Quantum information has produced surprising new insights into foundational questions about the anti-de Sitter/conformal field theory (AdS/CFT) correspondence, e.g., [1-11]. One fascinating concept that has recently entered this discussion is quantum circuit complexity: the size of the optimal unitary circuit or transformation $U_{T}$ preparing a target state $\left|\Psi_{T}\right\rangle$ from a given reference state $\left|\Psi_{R}\right\rangle$ using a set of elementary gates [12-14]. There have been a number of different proposals for the gravitational observables which would be dual to the complexity of a boundary state, e.g., [15-19]. The focus of our discussion will be the complexity $=$ action $(\mathrm{CA})$ conjecture $[17,18]$, which suggests

$$
\mathcal{C}_{A}(\Sigma)=I_{\mathrm{WDW}} / \pi
$$

That is, the holographic complexity of a boundary state on the time slice $\Sigma$ should be the gravitational action evaluated on the so-called Wheeler-DeWitt (WDW) patch, defined as the domain of dependence of a bulk spatial slice anchored on $\Sigma$. One important feature motivating the study of holographic complexity is that these new gravitational observables are sensitive to the bulk physics deep in the interior of a black hole $[15,20]$.

Exploring the properties of the new gravitational observables and their implications for complexity in the boundary theories is now an active area of research, e.g., [15-56].

Published by the American Physical Society under the terms of the Creative Commons Attribution 4.0 International license. Further distribution of this work must maintain attribution to the author(s) and the published article's title, journal citation, and DOI. Funded by SCOAP ${ }^{3}$.
A basic shortcoming of this research program is that we lack a proper understanding of circuit complexity of quantum field theories (QFTs). In particular, this prevents more than qualitative tests of the gravitational results. Hence, a second line of inquiry has become to fully develop the concept of circuit complexity for QFT states, in particular for states in a strongly coupled conformal field theory (CFT) (such as a holographic boundary theory), e.g., [57-84]. This will be essential to properly test the various holographic proposals and ultimately to produce a derivation of one (or more) of these conjectures. Our objective here is to begin to build a concrete bridge between these two research directions. In particular, we examine variations of the target state and demonstrate a natural interpretation connecting both approaches.

Nielsen's geometric approach [85-87] gives a framework to describe the complexity of QFT states, as illustrated for certain simple QFTs, e.g., [63-73]. It constructs a continuum representation of the unitary transformations

$$
U(\sigma)=\overleftarrow{\mathcal{P}} \exp \left(-i \int_{0}^{\sigma} d s H(s)\right)
$$

where $s$ parametrizes the circuit and $\overleftarrow{\mathcal{P}}$ signifies a right-toleft path ordering. The "Hamiltonian" $H(s)=\sum Y^{I}(s) \mathcal{O}_{I}$ is constructed from the (Hermitian) generators $\mathcal{O}_{I}$ of the elementary gates, and $Y^{I}(s)$ are control functions specifying which gates (and how many times they) are applied at any point $s$ in the circuit. Equation (2) actually specifies a path $U(\sigma)$ through the space of unitaries, or through the space of states with $|\psi(\sigma)\rangle=U(\sigma)\left|\psi_{R}\right\rangle$. With $\sigma \in[0,1]$, the boundary conditions are

$$
U(\sigma=0)=\mathbb{1}, \quad U(\sigma=1)=U_{T},
$$

where $\left|\Psi_{T}\right\rangle=U_{T}\left|\Psi_{R}\right\rangle$. 
Introducing a set of coordinates $x^{a}$ on the space of unitaries described by Eq. (2) [or on the space of states $\left.U\left(x^{a}\right)\left|\Psi_{R}\right\rangle\right]$, paths are described as $x^{a}(s)$. Nielsen's approach [85-87] identifies the optimal circuit producing $U_{T}$ by minimizing the cost

$$
\mathcal{D}\left[x^{a}(s)\right]=\int_{0}^{1} d s F\left(x^{a}, \dot{x}^{a}\right),
$$

subject to the boundary conditions (3). The circuit complexity is then the cost for the optimal trajectory, i.e.,

$$
\mathcal{C}\left(\left|\Psi_{T}\right\rangle\right) \equiv \operatorname{Min} \mathcal{D}
$$

As indicated in Eq. (4), the cost function $F$ is chosen as a particular functional of the position $x^{a}(s)$ and the tangent vector $\dot{x}^{a}(s)$. Hence, determining the complexity is analogous to the physical problem of identifying a particle trajectory by minimizing the action with Lagrangian $F\left(x^{a}, \dot{x}^{a}\right)$ and then evaluating the on-shell action. The precise form of $F$ is not fixed, but reasonable cost functions satisfy a number of preferred features [87]: (i) smoothness, (ii) positivity, (iii) triangle inequality, and (iv) positive homogeneity—see also $[63,65]$.

In the context of holography, we do not yet have a clear picture of the reference state, nor of the gates, nor of the path. However, the target state has a sharp interpretation in the AdS/CFT framework. Indeed, we are interested in quantum states in the boundary CFT which are dual to smooth geometries in the bulk gravitational theory. Hence, it is natural to examine the role of the latter in holographic complexity. In particular, we consider variations of the holographic complexity under changes of the target state and examine what information we can extract about the corresponding cost function.

The first law of complexity.-Using the analogy to classical mechanics, the variation of complexity (5) due to changing the target state with a fixed reference state, as illustrated in Fig. 1, yields

$$
\delta \mathcal{C}=\left.p_{a} \delta x^{a}\right|_{s=1} \quad \text { with } \quad p_{a}=\frac{\partial F}{\partial \dot{x}^{a}},
$$

for any differentiable cost function $F$. The significant feature of this result, which we designate as the first law of complexity, is that $\delta \mathcal{C}$ only has contributions from the end point. Hence, in the holographic setting, we can hope to

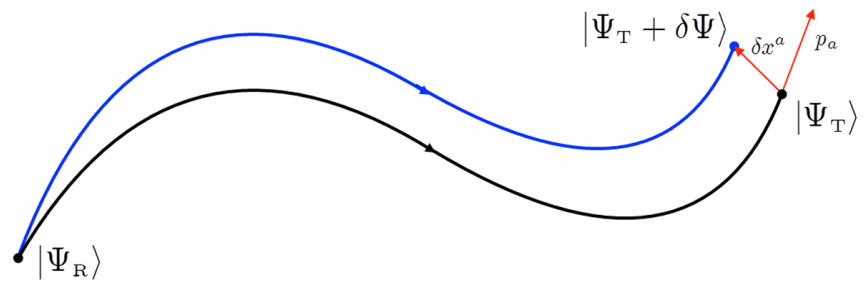

FIG. 1. The variation of the Nielsen circuit due to a perturbation $\left|\Psi_{T}+\delta \Psi\right\rangle$ of the target state $\left|\Psi_{T}\right\rangle$. extract information about the (variation of the) cost function in terms of bulk data describing the target state.

If the direction along the path $p_{a}$ is orthogonal to the variation of the target state $\delta x^{a}$, the first-order contribution (6) vanishes. However, the next order variation still comes from the end point,

$$
\begin{aligned}
\delta \mathcal{C} & =\left.\frac{1}{2} \delta p_{a} \delta x^{a}\right|_{s=1} \quad \text { with } \\
\delta p_{a} & =\delta x^{b} \frac{\partial^{2} F}{\partial x^{b} \partial \dot{x}^{a}}+\delta \dot{x}^{b} \frac{\partial^{2} F}{\partial \dot{x}^{b} \partial \dot{x}^{a}} .
\end{aligned}
$$

To explore the first law of complexity in the context of holography, we consider the $\mathrm{AdS}$ vacuum as our original target state and the backreaction of a free bulk scalar field with a small amplitude as the perturbed target state. Evaluating the variation of Eq. (1) yields the change in the corresponding holographic complexity. Now this excited state can be thought of as a coherent state of the bulk scalar. In particular, the scalar field can be expressed as

$$
\hat{\Phi}\left(y^{\mu}\right)=\sum\left[u_{n}\left(y^{\mu}\right) a_{n}+u_{n}^{*}\left(y^{\mu}\right) a_{n}^{\dagger}\right]
$$

where $u_{n}$ are eigenfunctions solving the Klein-Gordon equation in the AdS background. The $a_{n}$ and $a_{n}^{\dagger}$ denote the annihilation and creation operators acting on the scalar vacuum $|0\rangle$. We will assume that $y^{\mu}=(\rho, t, \Omega)$ denote global coordinates on the AdS background and then the sum over $n$ in Eq. (8) includes the radial and angular quantum numbers. The excited state in which a few modes $\{j\}$ are given a classical expectation value can be described as a coherent state

$\left|\varepsilon \alpha_{j}\right\rangle=e^{\varepsilon \sum D\left(\alpha_{j}\right)}|0\rangle \quad$ with $\quad D\left(\alpha_{j}\right)=\alpha_{j} a_{j}^{\dagger}-\alpha_{j}^{*} a_{j}$,

where we have included a small parameter $\varepsilon \ll 1$ to control the overall amplitude of the scalar field

$$
\left\langle\varepsilon \alpha_{j}|\hat{\Phi}| \varepsilon \alpha_{j}\right\rangle=\varepsilon \sum\left(\alpha_{j} u_{j}+\alpha_{j}^{*} u_{j}^{*}\right) \equiv \varepsilon \Phi_{\mathrm{cl}} .
$$

The reader will notice that our description of the perturbed state has been entirely in terms of the bulk theory while the aim of holographic complexity is to compute the complexity of states in the boundary theory. However, the AdS/CFT correspondence simply states that the bulk and boundary theories provide alternative descriptions of the same quantum states, i.e., the vacuum state $|0\rangle$ and the Hilbert space spanned with the $a_{n}$ and $a_{n}^{\dagger}$. Hence, while the details of the description change in terms of the boundary CFT, the perturbed states in Eq. (9) are still the same coherent states in the boundary theory. Further, $\delta \mathcal{C}_{A}$ is the variation of the complexity between these coherent states and the vacuum in the boundary theory. The bulk description of these states lends itself to the holographic calculations for $\delta \mathcal{C}_{A}$.

Holographic framework.-Our example begins with a four-dimensional bulk theory, Einstein gravity coupled to a negative cosmological constant, and a free massless scalar field 


$$
I_{\text {bulk }}=\frac{1}{16 \pi G_{N}} \int d^{4} y \sqrt{-g}\left(\mathcal{R}+\frac{6}{L^{2}}-\frac{1}{2} \nabla^{\mu} \Phi \nabla_{\mu} \Phi\right) .
$$

Its vacuum $\mathrm{AdS}_{4}$ solution is

$$
d s_{\text {AdS }}^{2}=\frac{L^{2}}{\cos ^{2} \rho}\left(-d t^{2}+d \rho^{2}+\sin ^{2} \rho d \Omega_{2}^{2}\right),
$$

where $L$ denotes the AdS radius of curvature and the (dimensionless) radial coordinate $\rho$ runs from 0 to $\pi / 2$, at the asymptotic boundary.

We perturb the vacuum by turning on the scalar in a coherent state (9) and the classical field $\Phi_{\mathrm{cl}}$ then backreacts on the spacetime geometry. Our calculation makes a perturbative expansion in $\varepsilon$ controlling the amplitude of the scalar in Eq. (10). While the full set of modes carry quantum numbers $n=(j, \ell, m)$, we focus on spherically symmetric configurations $\ell=m=0$. The scalar equation in the AdS background reduces to

$$
0=\partial_{\rho}^{2} \Phi+\frac{2}{\sin \rho \cos \rho} \partial_{\rho} \Phi-\partial_{t}^{2} \Phi
$$

The corresponding eigenfunctions in Eq. (8) are

$$
u_{j}(t, \rho)=e_{j}(\rho) e^{-i \omega_{j} t},
$$

with frequency $\omega_{j}=3+2 j$ and radial profile [88]

$$
\begin{aligned}
e_{j}(\rho) \equiv & 4(-)^{j} \sqrt{\frac{G_{N}(j+2)(j+1)}{\pi L^{2}\left(j+\frac{3}{2}\right)}} \\
& \times \cos ^{3} \rho_{2} F_{1}\left(-j, j+3 ; \frac{3}{2} ; \sin ^{2} \rho\right) .
\end{aligned}
$$

For simplicity, we will focus on real parameters $\alpha_{j}$ in Eq. (9) which then yields

$$
\Phi_{\mathrm{cl}}(t, \rho)=2 \sum \alpha_{j} e_{j}(\rho) \cos \left(\omega_{j} t\right)
$$

Next we turn to the backreaction, where we follow closely the analysis in [106-108]. For our spherically symmetric configurations (16), we use the metric ansatz

$$
d s^{2}=\frac{L^{2}}{\cos ^{2} \rho}\left(-a e^{-2 d} d t^{2}+\frac{d \rho^{2}}{a}+\sin ^{2} \rho d \Omega_{2}^{2}\right),
$$

where $a(t, \rho), d(t, \rho)$ describe the metric perturbation. Working in the small amplitude expansion, we write

$$
\begin{aligned}
a(t, \rho) & =1+\varepsilon^{2} a_{2}(t, \rho)+\mathcal{O}\left(\varepsilon^{4}\right), \\
e^{d(t, \rho)} & =1+\varepsilon^{2} d_{2}(t, \rho)+\mathcal{O}\left(\varepsilon^{4}\right),
\end{aligned}
$$

and at $\mathcal{O}\left(\varepsilon^{2}\right)$, Einstein's equations reduce to three linear first-order differential equations

$$
\begin{aligned}
& \partial_{\rho} a_{2}+\frac{3-2 \cos ^{2} \rho}{\cos \rho \sin \rho} a_{2}=\partial_{\rho} d_{2}, \\
& \partial_{\rho} d_{2}=-\frac{1}{4} \sin \rho \cos \rho\left[\left(\partial_{\rho} \Phi_{\mathrm{cl}}\right)^{2}+\left(\partial_{t} \Phi_{\mathrm{cl}}\right)^{2}\right], \\
& \partial_{t} a_{2}=-\frac{1}{2} \sin \rho \cos \rho \partial_{\rho} \Phi_{\mathrm{cl}} \partial_{t} \Phi_{\mathrm{cl}},
\end{aligned}
$$

with the third being redundant. Imposing the regularity condition $a_{2}(t, \rho=0)=0$ and the boundary condition $d_{2}(t, \rho=\pi / 2)=0$, the perturbations $a_{2}(t, \rho)$ and $d_{2}(t, \rho)$ can be integrated in terms of $\Phi_{\mathrm{cl}}(t, \rho)[107,108]$.

Holographic complexity.-The variation of holographic complexity evaluated to second order in $\varepsilon$ by the CA conjecture (1) splits into two classes of contributions

$$
\delta \mathcal{C}_{A}(\Sigma)=\frac{1}{\pi}\left(\delta I_{\mathrm{WDW}}+I_{\delta \mathrm{WDW}}\right)
$$

where $\delta I_{\mathrm{WDW}}$ is the variation due to the change in the background fields within the original WDW patch, while $I_{\delta \mathrm{WDW}}$ is the variation due to the change in the shape (i.e., the position of the boundary) of the WDW patch.

First, we must recall that as well as the bulk terms appearing in Eq. (11), the gravitational action includes a number of surface terms [24,109]. In the present case [88], only two will be relevant in evaluating the variation (20): the first is the null surface term

$$
I_{\kappa}=\frac{1}{8 \pi G_{N}} \int_{\partial \mathrm{WDW}} d s d^{2} \Omega \sqrt{\gamma} \kappa,
$$

where $\gamma$ is the metric determinant on the boundary of the WDW patch. $\kappa$ describes the failure of the coordinate $s$ along the null boundary to be affine, i.e., $k^{\mu} \nabla_{\mu} k_{\nu}=\kappa k_{\nu}$, where $k_{\mu} d x^{\mu}$ is the outward-directed null normal. The second term

$$
I_{\mathrm{ct}}=\frac{1}{8 \pi G_{N}} \int_{\partial \mathrm{wDW}} d s d^{2} \Omega \sqrt{\gamma} \Theta \log \left(\ell_{\mathrm{ct}} \Theta\right)
$$

ensures the action is invariant under reparametrizations of the null boundaries [24]. Here, $\Theta=\partial_{s} \log \sqrt{\gamma}$ is the expansion scalar of the null generators on the boundary, and $\ell_{\mathrm{ct}}$ is an arbitrary scale needed for the argument of the logarithm to be dimensionless.

If we consider the WDW patch anchored at $t=0$ in the AdS vacuum (12), the future and past null boundaries are given by $t=t_{ \pm}(\rho)= \pm(\pi / 2-\rho)$, and we choose the null normals as $k_{\mu} d x^{\mu}= \pm d t+d \rho$. The boundary coordinate is implicitly defined by $\partial_{s} \equiv k^{\mu} \partial_{\mu}=\cos ^{2} \rho / L^{2}\left(\mp \partial_{t}+\partial \rho\right)$.

In the perturbed background, the null boundaries experience a small shift $\delta t_{ \pm}(\rho)$, which is determined by

$$
\partial_{\rho} \delta t_{ \pm}= \pm\left.\varepsilon^{2}\left(a_{2}-d_{2}\right)\right|_{t=t_{ \pm}(\rho)} .
$$

The variation $\delta I_{\text {bulk }}$ contributes to $I_{\delta \text { WDW }}$ in Eq. (20) by the boundary integrals of $\delta t_{ \pm}(\rho)$ times the bulk action evaluated for the AdS vacuum, i.e., $\mathcal{R}+6 / L^{2}=-6 / L^{2}$, and to $\delta I_{\text {WDW }}$ yielding a total derivative, which is evaluated 
as a surface integral on the undeformed boundaries $t=t_{ \pm}(\rho)$. Hence, the entire variation $\delta \mathcal{C}_{A}(\Sigma)$ is given by surface integrals along the boundary of the WDW patch.

Turning to the contributions coming from Eqs. (21) and (22). Above, we chose an affine boundary coordinate $s$, giving $\kappa=0$ at leading order. The simplest approach is to keep the same coordinate for the second-order calculations and, at this order, it fails to be affine. Hence, we have a nonvanishing variation $\delta I_{\kappa}$ with

$$
\kappa= \pm \varepsilon^{2} \frac{\cos ^{2} \rho}{L^{2}} \partial_{t}\left(a_{2}-d_{2}\right) .
$$

The variation $\delta I_{\mathrm{ct}}$ reduces to

$$
\delta I_{\mathrm{ct}}=\frac{1}{8 \pi G_{N}} \int_{\partial \mathrm{WDW}} d s d^{2} \Omega \delta k^{\mu} \partial_{\mu} \sqrt{\gamma} .
$$

We note that this result is independent of the scale $\ell_{\mathrm{ct}}$.

Combining all contributions, the holographic complexity variation equals

$\delta \mathcal{C}_{A}(\Sigma)=\frac{\delta I_{\mathrm{mat}}}{\pi}=-\frac{\varepsilon^{2}}{64 \pi^{2} G_{N}} \int_{\partial \mathrm{WDW}} d s d^{2} \Omega \sqrt{\gamma} \partial_{s}\left(\Phi_{\mathrm{cl}}^{2}\right)$.

Above we are emphasizing that the sum of all gravitational contributions precisely cancels and the full variation comes entirely from the variation of the scalar field action $\delta I_{\text {mat }}$. Given the state in Eq. (9), this becomes

$$
\delta \mathcal{C}_{A}(\Sigma)=\frac{\varepsilon^{2}}{\pi^{2}} \sum_{j_{1}, j_{2}} \alpha_{j_{1}} \alpha_{j_{2}} C_{j_{1}, j_{2}},
$$

with the coefficients given by

$$
\begin{aligned}
C_{j_{1}, j_{2}}= & \sqrt{\frac{\left(j_{1}+\frac{3}{2}\right)\left(j_{2}+\frac{3}{2}\right)}{\left(j_{1}+1\right)\left(j_{1}+2\right)\left(j_{2}+1\right)\left(j_{2}+2\right)}} \\
& \times\left(H_{j_{1}+\frac{1}{2}}+H_{j_{1}+\frac{3}{2}}+H_{j_{2}+\frac{1}{2}}+H_{j_{2}+\frac{3}{2}}\right. \\
& \left.-H_{j_{1}+j_{2}+\frac{5}{2}}-H_{j_{1}-j_{2}-\frac{1}{2}}-2+4 \log 2\right) .
\end{aligned}
$$

The $H_{\alpha}=\partial_{\alpha} \log \Gamma(\alpha+1)+\gamma_{0}$ are harmonic numbers (where $\gamma_{0}$ is Euler's constant). The reflection relation $H_{-\alpha-1}-H_{\alpha}=\pi \cot (\pi \alpha)$ guarantees $C_{j_{1}, j_{2}}=C_{j_{2}, j_{1}}$.

Figure 2 shows $C_{j_{1}, j_{2}}$ as a function of $j_{1}$ for various values of $j_{2}$. We can see that these curves have two peaks, one at $j_{1}=1$ and the other at $j_{1}=j_{2}$. However, in both instances, the peak value decays as $j_{2}$ grows. In fact, near the diagonal peak, one has

$$
\lim _{j \rightarrow \infty} C_{j, j+\delta j}=3 \frac{\log 2 j}{j}+\mathcal{O}\left(\frac{1}{j}\right),
$$

with fixed $\delta j$. This further indicates that the curves are relatively flat for large $j$ since $\delta j$ only appears in the $\mathcal{O}(1 / j)$ term. We might also note that for large $j_{1}$ (and fixed $j_{2}$ ), the curves are decaying as $\left(j_{1}-j_{2}\right)^{-1 / 2}$.

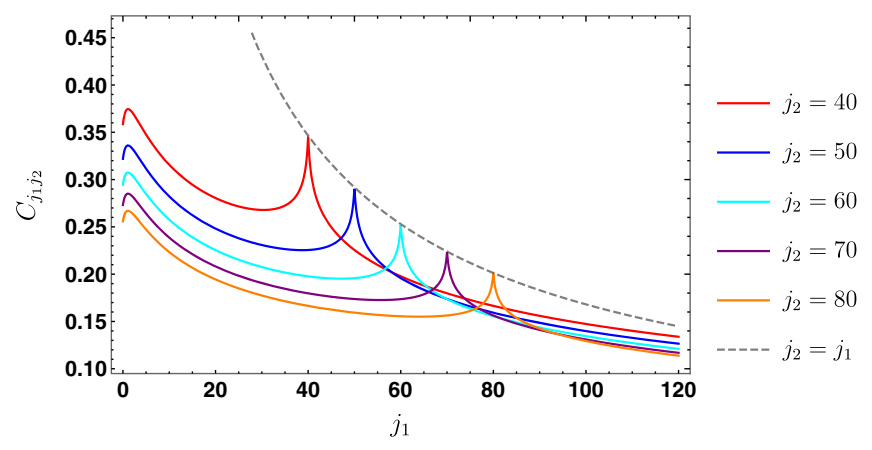

FIG. 2. The plot for $C_{j_{1}, j_{2}}$ with fixed $j_{2}$. Each curve has peaks at $j_{1}=1$ and $j_{1}=j_{2}$. The envelope of the latter is shown with the dashed gray line. Although we draw continuous curves to help guide the eye, one should only think of $j_{1}$ as taking integer values, i.e., $j_{1}=0,1,2, \ldots$.

Discussion.-We applied the first law of complexity to examine variations of holographic complexity generated by a small scalar field excitation in AdS. Our results in Eqs. (27) and (28) depend only on the dimensionless parameters, $\varepsilon$ and $\alpha_{j}$, characterizing these excitations (9). These are coherent states of the bulk scalar, but are equally described as coherent states of the dual marginal operator (and its descendants) in the boundary theory. Hence, these parameters and $\delta \mathcal{C}_{A}$ have a natural interpretation as boundary quantities. It is interesting that $\delta \mathcal{C}_{A}$ is scale independent, in contrast to the full holographic complexity, e.g., where the leading UV divergence has the form $\mathcal{C}_{A} \sim \log \left(2 \ell_{\mathrm{ct}} / L\right) \operatorname{Vol}(\Sigma) / \delta^{2}$ with $\delta$ being the short-distance cutoff $[28,110]$.

Given that our final result (27) is second order, i.e., $\delta \mathcal{C}_{A} \sim \varepsilon^{2} \alpha^{2}$, the first-order variation $p_{a} \delta x^{a}$ in Eq. (6) must vanish. That is, the cost function appropriate for the CA conjecture defines a geometry where the coherent state directions are orthogonal to the direction along the circuit preparing the CFT vacuum. Instead, the leading variation of the holographic complexity in this example takes the form given in Eq. (7).

It is difficult to interpret this result without further assumptions. For example, let us assume that the cost function has the simple form $F=g_{a b} \dot{x}^{a} \dot{x}^{b}$, known as the $\kappa=2$ measure [67]. Then the vanishing of the first-order variation indicates that at the end point of the circuit, the off-diagonal components of the metric $g_{a b}$ between the coherent state and vacuum preparation directions vanish. If this vanishing holds in the vicinity of the end point, i.e., it also holds for the first derivative of the metric, then the remaining variation of the complexity takes the form $\delta \mathcal{C}=g_{a b} \delta x^{a} \delta \dot{x}^{b}$. In this scenario, comparing to Eq. (27), the coefficients can be interpreted directly as metric components on the corresponding space, i.e., $C_{j_{1}, j_{2}} \sim g_{j_{1} j_{2}}$.

We can compare our holographic results to the variation of the complexity for a free massless scalar field in a fixed AdS geometry (12) by evaluating the circuit complexity of the vacuum state and the coherent states (9) following 
$[63,67]$. In this setup, the reference state is an unentangled state of local scalar field degrees of freedom in the AdS space, while in holography, it corresponds to an unentangled state of quantum gravity degrees of freedom (and so presumably there is no spacetime). The circuit complexity in the QFT depends on the choice of the cost function (see $[67,89]$ for further details); however, a characteristic result for the $\kappa=2$ measure is

$$
\delta \mathcal{C}_{\kappa=2}=\sum \frac{2 \varepsilon^{2} \alpha_{n}^{2}}{\mu^{2} x_{0}^{2}\left(\omega_{n} / R \mu-1\right)} \log \left(\frac{\omega_{n}}{R \mu}\right),
$$

where $\omega_{n}=3+2 j+\ell$ is the eigenfrequency for modes with $n=(j, \ell, m), \mu$ is the frequency characterizing the reference state, $x_{0}$ is a scale appearing in the definition of the gates producing the coherent state [67], and $R$ is an additional length scale introduced to produce a dimensionful time in the metric (12). This QFT variation is second order; i.e., the coherent state directions are orthogonal to the direction of the circuit preparing the QFT vacuum, as in our holographic framework. Its large radial quantum number limit, i.e., $\omega_{n} \sim 2 j$ matches the large $j$ limit of the holographic result given in Eq. (29). In contrast to the holographic result, all the coherent state directions are mutually orthogonal due to the orthogonality of the scalar modes (14) making Eq. (30) diagonal (with $j=j_{1}=j_{2}$ ). Furthermore, the absence of scales in the holographic result would require the QFT scales to be dependent, e.g., $\mu x_{0} \sim 1 \sim \mu R$.

There is an important assumption in our derivation of the first law of complexity. When the complexity (5) is described as the minimal cost of circuits preparing the desired target state, we mean the global minimum over all possible circuits. When we perturb the target state, we assume that the circuit which globally minimizes the cost remains close to the original optimal circuit; i.e., the family of globally minimizing circuits is continuous in the amplitude of the perturbation. While one can imagine examples where this is not the case (e.g., geodesics between "nearly" conjugate points on a sphere), our expectation is that this assumption is valid for the example studied here. In particular, it is explicitly seen in the QFT complexity calculations [67]. Of course, it would also be interesting to identify situations (in either QFT or holography) where our assumption does not hold.

Returning to the holographic calculations, we recall the inclusion of the counterterm (22) was essential for the cancellation of the gravitational contributions to the action variation leading to $\delta \mathcal{C}_{A}$ entirely determined by the scalar field contributions. This feature may add to the essential role of this boundary term for the CA proposal [43,110], despite not being necessary to have a well-defined variational principle for the gravitational action [24]. It would be interesting to better understand this cancellation and how generally it applies, e.g., does it hold beyond spherical symmetry.

Irrespective of the previous cancellation, another feature of our calculations was that all of the contributions reduced to surface contributions on the boundaries of the WDW patch. In analogy to the derivation of our first law, this property essentially arises because we are considering variations of the bulk action evaluated around background on-shell configurations. In the case of the Nielsen geometry, the boundary contribution comes from the (target state) end of the circuit, e.g., see Fig. 1. Hence, we are led to speculate that the boundary of the WDW patch may correspond to the "end of the circuit" in the CA conjecture. This suggests a picture where the AdS spacetime is built up through adding layers of null cones. This interpretation may have connections with the surface or state correspondence of [111].

The first law of complexity provides a new approach to investigate holographic complexity and in particular, to build a concrete bridge to standard approaches to circuit complexity. While we have provided one application of this method here, this is only a starting point. It will be straightforward to extend our calculations to other fields (e.g., massive scalars or gravitons), higher spacetime dimensions, or other quantum states. The same approach can also be used to investigate the complexity $=$ volume $[15,16]$ and complexity = spacetime volume [19] conjectures. While we initially assumed that complexity is defined by a Nielsen geometry, a similar extremization arises in the Fubini-Study approach of [64] and in the path integral optimization procedure of [59-62]. Hence, our approach should be useful to investigate these directions as well. More generally, it provides a unified perspective with which to investigate variations of holographic complexity, e.g., see [55-59]. We will explore several of these questions in [89].

It is a pleasure to thank Alex Belin, Alex Buchel, Pawel Caputa, Horacio Casini, Vincent Chen, Jordan Cotler, José M. Figueroa-O'Farrill, Ling-Yan Hung, Javier Magan, Alex Maloney, Hugo Marrochio, James Sully, Tadashi Takayanagi, and Jingxiang Wu for useful comments and conversations. Research at Perimeter Institute is supported by the Government of Canada through the Department of Innovation, Science and Economic Development, and by the Province of Ontario through the Ministry of Economic Development, Job Creation and Trade. A. B. acknowledges support by the program "Rita Levi Montalcini" for your young researchers and the INFN initiative GAST. J. H. and S.M.R. are supported in part by a Discovery Grant awarded to RCM by the Natural Sciences and Engineering Research Council of Canada. J. H. is also supported by the Natural Sciences and Engineering Research Council of Canada through a NSERC PGS-D. R. C. M. was supported in part by research funding from the Simons Foundation through the "It from Qubit" Collaboration and from the BMO Financial Group. J. S. is supported by the Science and Technology Facilities Council (Grant No. ST/L000458/1]. J. S. would also like to thank the Perimeter Institute for all their support and 
hospitality when this project started. A. B., F.G., and R.C.M. thank the Galileo Galilei Institute for Theoretical Physics for hospitality and the INFN for partial support during part of this work.

*abernamonti@perimeterinstitute.ca

†fgalli@perimeterinstitute.ca

\#hernandez@ perimeterinstitute.ca

${ }^{\S}$ rmyers@perimeterinstitute.ca

" sruan@perimeterinstitute.ca

†.simon@ed.ac.uk

[1] S. Ryu and T. Takayanagi, Phys. Rev. Lett. 96, 181602 (2006).

[2] S. Ryu and T. Takayanagi, J. High Energy Phys. 08 (2006) 045.

[3] V. E. Hubeny, M. Rangamani, and T. Takayanagi, J. High Energy Phys. 07 (2007) 062.

[4] M. Rangamani and T. Takayanagi, Lect. Notes Phys. 931, 1 (2017).

[5] B. Swingle, Phys. Rev. D 86, 065007 (2012).

[6] R. C. Myers and A. Sinha, J. High Energy Phys. 01 (2011) 125.

[7] D. D. Blanco, H. Casini, L.-Y. Hung, and R. C. Myers, J. High Energy Phys. 08 (2013) 060.

[8] X. Dong, J. High Energy Phys. 01 (2014) 044.

[9] T. Faulkner, M. Guica, T. Hartman, R. C. Myers, and M. Van Raamsdonk, J. High Energy Phys. 03 (2014) 051.

[10] A. Almheiri, X. Dong, and D. Harlow, J. High Energy Phys. 04 (2015) 163.

[11] F. Pastawski, B. Yoshida, D. Harlow, and J. Preskill, J. High Energy Phys. 06 (2015) 149.

[12] S. Aaronson, arXiv:1607.05256.

[13] J. Watrous, Encyclopedia of Complexity and Systems Science (Springer, New York, 2009), pp. 7174-7201.

[14] L. Susskind, arXiv:1810.11563.

[15] L. Susskind, Fortschr. Phys. 64, 24 (2016).

[16] D. Stanford and L. Susskind, Phys. Rev. D 90, 126007 (2014).

[17] A. R. Brown, D. A. Roberts, L. Susskind, B. Swingle, and Y. Zhao, Phys. Rev. Lett. 116, 191301 (2016).

[18] A. R. Brown, D. A. Roberts, L. Susskind, B. Swingle, and Y. Zhao, Phys. Rev. D 93, 086006 (2016).

[19] J. Couch, W. Fischler, and P. H. Nguyen, J. High Energy Phys. 03 (2017) 119.

[20] L. Susskind, Fortschr. Phys. 64, 49 (2016).

[21] L. Susskind and Y. Zhao, arXiv:1408.2823.

[22] L. Susskind, Fortschr. Phys. 64, 84 (2016).

[23] D. A. Roberts, D. Stanford, and L. Susskind, J. High Energy Phys. 03 (2015) 051.

[24] L. Lehner, R. C. Myers, E. Poisson, and R. D. Sorkin, Phys. Rev. D 94, 084046 (2016).

[25] R.-G. Cai, S.-M. Ruan, S.-J. Wang, R.-Q. Yang, and R.-H. Peng, J. High Energy Phys. 09 (2016) 161.

[26] A. Reynolds and S. F. Ross, Classical Quantum Gravity 34, 105004 (2017).

[27] S. Chapman, H. Marrochio, and R. C. Myers, J. High Energy Phys. 01 (2017) 062.
[28] D. Carmi, R. C. Myers, and P. Rath, J. High Energy Phys. 03 (2017) 118.

[29] M. Moosa, J. High Energy Phys. 03 (2018) 031.

[30] J. Couch, S. Eccles, W. Fischler, and M.-L. Xiao, J. High Energy Phys. 03 (2018) 108.

[31] R.-G. Cai, M. Sasaki, and S.-J. Wang, Phys. Rev. D 95, 124002 (2017).

[32] A. R. Brown and L. Susskind, Phys. Rev. D 97, 086015 (2018).

[33] D. Carmi, S. Chapman, H. Marrochio, R. C. Myers, and S. Sugishita, J. High Energy Phys. 11 (2017) 188.

[34] B. Swingle and Y. Wang, J. High Energy Phys. 09 (2018) 106.

[35] M. Flory, J. High Energy Phys. 06 (2017) 131.

[36] Y. Zhao, Phys. Rev. D 97, 126007 (2018).

[37] R. Abt, J. Erdmenger, H. Hinrichsen, C. M. MelbyThompson, R. Meyer, C. Northe, and I. A. Reyes, Fortschr. Phys. 66, 1800034 (2018).

[38] R. Abt, J. Erdmenger, M. Gerbershagen, C. M. MelbyThompson, and C. Northe, J. High Energy Phys. 01 (2019) 012.

[39] M. Alishahiha, A. Faraji Astaneh, M. R. Mohammadi Mozaffar, and A. Mollabashi, J. High Energy Phys. 07 (2018) 042.

[40] Y.-S. An and R.-H. Peng, Phys. Rev. D 97, 066022 (2018).

[41] Z. Fu, A. Maloney, D. Marolf, H. Maxfield, and Z. Wang, J. High Energy Phys. 02 (2018) 072.

[42] S. Mahapatra and P. Roy, J. High Energy Phys. 11 (2018) 138.

[43] S. Chapman, H. Marrochio, and R. C. Myers, J. High Energy Phys. 06 (2018) 046.

[44] S. Chapman, H. Marrochio, and R. C. Myers, J. High Energy Phys. 06 (2018) 114.

[45] P. A. Cano, R. A. Hennigar, and H. Marrochio, Phys. Rev. Lett. 121, 121602 (2018).

[46] J. L. F. Barbon and J. Martin-Garcia, J. High Energy Phys. 06 (2018) 132.

[47] L. Susskind, arXiv:1802.02175.

[48] L. Susskind, arXiv:1802.01198.

[49] S. Cooper, M. Rozali, B. Swingle, M. Van Raamsdonk, C. Waddell, and D. Wakeham, J. High Energy Phys. 07 (2019) 065.

[50] T. Numasawa, arXiv:1811.03597.

[51] A. R. Brown, H. Gharibyan, A. Streicher, L. Susskind, L. Thorlacius, and Y. Zhao, Phys. Rev. D 98, 126016 (2018).

[52] K. Goto, H. Marrochio, R. C. Myers, L. Queimada, and B. Yoshida, J. High Energy Phys. 02 (2019) 160.

[53] C. A. Agón, M. Headrick, and B. Swingle, J. High Energy Phys. 02 (2019) 145.

[54] S. Chapman, D. Ge, and G. Policastro, J. High Energy Phys. 05 (2019) 049.

[55] M. Flory and N. Miekley, J. High Energy Phys. 05 (2019) 003.

[56] M. Flory, J. High Energy Phys. 05 (2019) 086.

[57] A. Belin, A. Lewkowycz, and G. Sárosi, J. High Energy Phys. 03 (2019) 044.

[58] A. Belin, A. Lewkowycz, and G. Sárosi, Phys. Lett. B 789, 71 (2019). 
[59] A. Bhattacharyya, P. Caputa, S. R. Das, N. Kundu, M. Miyaji, and T. Takayanagi, J. High Energy Phys. 07 (2018) 086.

[60] T. Takayanagi, J. High Energy Phys. 12 (2018) 048.

[61] P. Caputa, N. Kundu, M. Miyaji, T. Takayanagi, and K. Watanabe, Phys. Rev. Lett. 119, 071602 (2017).

[62] P. Caputa, N. Kundu, M. Miyaji, T. Takayanagi, and K. Watanabe, J. High Energy Phys. 11 (2017) 097.

[63] R. A. Jefferson and R. C. Myers, J. High Energy Phys. 10 (2017) 107.

[64] S. Chapman, M. P. Heller, H. Marrochio, and F. Pastawski, Phys. Rev. Lett. 120, 121602 (2018).

[65] L. Hackl and R. C. Myers, J. High Energy Phys. 07 (2018) 139.

[66] R. Khan, C. Krishnan, and S. Sharma, Phys. Rev. D 98, 126001 (2018).

[67] M. Guo, J. Hernandez, R. C. Myers, and S.-M. Ruan, J. High Energy Phys. 10 (2018) 011.

[68] A. Bhattacharyya, A. Shekar, and A. Sinha, J. High Energy Phys. 10 (2018) 140.

[69] S. Chapman, J. Eisert, L. Hackl, M. P. Heller, R. Jefferson, H. Marrochio, and R.C. Myers, SciPost Phys. 6, 034 (2019).

[70] D. W. F. Alves and G. Camilo, J. High Energy Phys. 06 (2018) 029.

[71] H. A. Camargo, P. Caputa, D. Das, M. P. Heller, and R. Jefferson, arXiv:1807.07075.

[72] T. Ali, A. Bhattacharyya, S. Shajidul Haque, E. H. Kim, and N. Moynihan, J. High Energy Phys. 04 (2019) 087.

[73] J. Jiang and X. Liu, Phys. Rev. D 99, 026011 (2019).

[74] D. A. Roberts and B. Yoshida, J. High Energy Phys. 04 (2017) 121.

[75] K. Hashimoto, N. Iizuka, and S. Sugishita, Phys. Rev. D 96, 126001 (2017).

[76] B. Czech, Phys. Rev. Lett. 120, 031601 (2018).

[77] A. Reynolds and S. F. Ross, Classical Quantum Gravity 34, 175013 (2017).

[78] J. M. Magan, J. High Energy Phys. 09 (2018) 043.

[79] P. Caputa and J. M. Magan, Phys. Rev. Lett. 122, 231302 (2019).

[80] V. Balasubramanian, M. DeCross, A. Kar, and O. Parrikar, J. High Energy Phys. 02 (2019) 069.

[81] R. Jackiw, Nucl. Phys. B252, 343 (1985).

[82] T. Shimaji, T. Takayanagi, and Z. Wei, J. High Energy Phys. 03 (2019) 165.

[83] T. Ali, A. Bhattacharyya, S. Shajidul Haque, E. H. Kim, and N. Moynihan, arXiv:1811.05985.

[84] F. Liu, R. Lundgren, P. Titum, J. R. Garrison, and A. V. Gorshkov, arXiv:1902.10720.

[85] M. A. Nielsen, M. R. Dowling, M. Gu, and A. C. Doherty, Science 311, 1133 (2006).

[86] M. R. Dowling and M. A. Nielsen, Quantum Inf. Comput. 8, 861 (2008).

[87] M. A. Nielsen, arXiv:quant-ph/0502070.
[88] See Supplemental Material http://link.aps.org/ supplemental/10.1103/PhysRevLett.123.081601 for more details, which include Refs. [89-105].

[89] A. Bernamonti, F. Galli, J. Hernandez, R. C. Myers, S.-M. Ruan, and J. Simón (to be published).

[90] Hence, the standard normalization for a scalar in QFT calculations is achieved by defining as $\Psi=\Phi / \sqrt{16 \pi G_{N}}$.

[91] S. J. Avis, C. J. Isham, and D. Storey, Phys. Rev. D 18, 3565 (1978).

[92] C. P. Burgess and C. A. Lutken, Phys. Lett. 153B, 137 (1985).

[93] I. I. Cotăescu, Phys. Rev. D 60, 107504 (1999).

[94] A. L. Fitzpatrick and J. Kaplan, arXiv:1104.2597.

[95] J. Kaplan, Lectures on AdS/CFT from the bottom up, (2013).

[96] S. Terashima, J. High Energy Phys. 02 (2018) 019.

[97] M. Ammon and J. Erdmenger, Gauge/Gravity Duality (Cambridge University Press, Cambridge, United Kingdom, 2015).

[98] S. El-Showk and K. Papadodimas, J. High Energy Phys. 10 (2012) 106.

[99] M. Botta-Cantcheff, P. Martínez, and G. A. Silva, J. High Energy Phys. 02 (2016) 171.

[100] D. Marolf, O. Parrikar, C. Rabideau, A. Izadi Rad, and M. Van Raamsdonk, J. High Energy Phys. 06 (2018) 077.

[101] M. Botta-Cantcheff, P. J. Martínez, and G. A. Silva, J. High Energy Phys. 04 (2019) 028.

[102] No horizon is expected for a perturbative excitation of the AdS spacetime, but technically the possibility of opening an event horizon is removed by the boundary condition $a_{2}(t, \rho=0)=0$, which corresponds to the geometry closing off smoothly at the origin of the radius.

[103] J. W. York, Phys. Rev. Lett. 28, 1082 (1972).

[104] G. W. Gibbons and S. W. Hawking, Phys. Rev. D 15, 2752 (1977).

[105] The first equality can be verified writing the integrals explicitly in terms of the radial variable and noticing that within our perturbative setup, $d s=\left(d \rho / k_{0}^{\rho}\right)\left(1-\delta k^{\rho}\right)$ on the boundary of the deformed WDW patch.

[106] P. Bizon and A. Rostworowski, Phys. Rev. Lett. 107, 031102 (2011).

[107] A. Buchel, L. Lehner, and S. L. Liebling, Phys. Rev. D 86, 123011 (2012).

[108] A. Buchel, S. L. Liebling, and L. Lehner, Phys. Rev. D 87, 123006 (2013).

[109] The scalar field action has a well-defined variational principle without any additional surface terms, and so we assume no surface terms are added for the scalar.

[110] S. Chapman, H. Marrochio, and R. C. Myers, J. High Energy Phys. 06 (2018) 114.

[111] M. Miyaji and T. Takayanagi, Prog. Theor. Exp. Phys. (2015), 073B03. 